\title{
Dipole-Mode Vector Solitons
}

\author{
Juan J. García-Ripoll, ${ }^{1}$ Víctor M. Pérez-García, ${ }^{1}$ Elena A. Ostrovskaya, ${ }^{2}$ and Yuri S. Kivshar ${ }^{2}$ \\ ${ }^{1}$ Departamento de Matemáticas, Escuela Técnica Superior de Industriales, Universidad de Castilla-La Mancha \\ 13071 Ciudad Real, Spain \\ ${ }^{2}$ Optical Sciences Centre, The Australian National University, Canberra ACT 0200, Australia
}

(Received 20 December 1999)

\begin{abstract}
We find a new type of optical vector soliton that originates from trapping of a dipole mode by the soliton-induced waveguides. These solitons, which appear as a consequence of the vector nature of the two-component system, are more stable than the previously found optical vortex solitons and represent a new type of extremely robust nonlinear vector structure.
\end{abstract}

PACS numbers: 42.65.Tg, 05.45.Yv, 42.65.Sf

Perhaps one of the most desirable goals of optics is the development of purely optical devices in which light can be used to guide and manipulate light itself. This motivation explains the growing interest in self-guided beams (or spatial optical solitons) and the recent theoretical and experimental study of spatial solitons and their interactions [1]. Not only reconfigurable and steerable soliton-induced waveguides created in a bulk medium are of particular practical interest. Indeed, a spatial soliton that guides another beam (of a different polarization or frequency) may become a new object, a vector soliton [2], with an internal structure and new dynamical properties which yield surprising results for the stability of such an object even in the simplest cases.

Complex phenomena induced by the vector nature of the nonlinear wave equations arise in many fields of physics and are already classical in the realm of condensed matter physics, dynamics of biomolecules, nonlinear optics, etc. Recently an interest in these complex structures and the theoretical possibilities they offer has been renewed because of their experimental realization in different physical contexts. For instance, vector phenomena have been observed in Bose-Einstein condensation, with vortices in multicomponent condensates [3] or nontrivial topological defects due to interspecies interaction [4]. Finally, vectorial active optical media are also being currently investigated because of the many new characteristics they provide, as compared to scalar systems [5].

In this Letter we study a vector object formed by two optical beams that interact incoherently in a bulk nonlinear medium. If the nonlinearity of the medium is selffocusing, an isolated beam, under proper conditions, will form a self-trapped state-a spatial soliton [1]. Such a spatial soliton changes the refractive index of the medium and creates a stationary effective waveguide. A second beam of a much lower intensity is subjected to the induced change of the refractive index and can be trapped as a localized mode of that waveguide.

From the linear optical waveguide theory, we expect that a radially symmetric waveguide can host different types of modes with more elaborate geometries [Figs. 1(a)-1(c)].
However, at higher intensities of the trapped beam, one must regard the two beams as components of a vector soliton, self-trapped by a self-consistent change of the refractive index induced by both beams. In this case we may not treat the shapes of the beams as independent and it is not trivial to conclude whether we may obtain states which are a composition of a lowest-order state in one component and a high-order state in the other one.

Recalling a previous work on two-component onedimensional solitons [6], where stable multihump configurations were obtained, one may expect at least two types of such complex objects in the two-dimensional case. The first generalization is the recently discussed two-dimensional vector soliton $[7,8]$ which has a nodeless shape [e.g., as shown in Fig. 1(a)] in the first component and a vortex in the second one [Fig. 1(b)]. The second generalization introduced here maintains a similar shape for the first component, while the second beam develops a node along a certain direction [Fig. 1(c)], forming what we call a dipole-mode soliton.

The purpose of this Letter is twofold. First, we discuss the stability of the vortex vector solitons and show that these objects are linearly unstable and decay into dipole-mode vector solitons. Second, we prove that these dipole-mode solitons exist for an ample range of relative intensities of both components and show that they survive both small and large amplitude perturbations, their propagation dynamics resembling that of two spiraling beams [9].

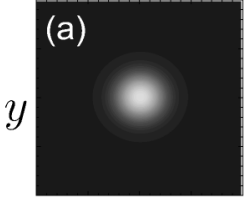

$x$

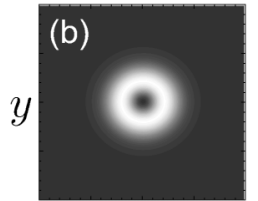

$x$

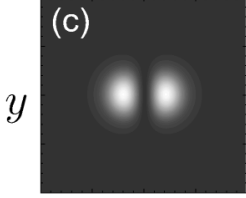

$x$
FIG. 1. Intensity distribution of (a) fundamental, (b) vortex, and (c) dipole modes guided by a radially symmetric fundamental soliton. 
We would like to emphasize here that both results are highly nontrivial. While it is commonly believed that asymmetric solitary waves possess a higher energy, and should be a priori unstable, our results demonstrate that the opposite is true: An excited state with an elaborate geometry may indeed be more stable than a radially symmetric one and, as such, would be a better candidate for experimental realization. To this point, we must remark that the recently discovered method of creating multicomponent solitons in a photorefractive medium [10] would allow a simple and direct verification of our theory, including the questions of soliton existence and stability.

The outline of this paper is as follows. We formulate the model for our system and lay out the equations. A reasonable range of the only dimensionless parameter of the model is established and we proceed with the study of vortex-mode vector solitons in that range. We recall previous studies on the issue of the existence of such states $[7,8]$ and add new results arising from a linear stability analysis and numerical simulations of the dynamics of these solitons. Having concluded that the vortex-mode vector solitons are linearly unstable, we proceed with the study of the dipole variant. We obtain a continuous family of solutions in the same parameter range. In this case, our tools for the linear stability analysis give no conclusive results, but numerical simulations of highly perturbed states show a periodic, stable evolution. Finally we conclude and summarize the implications of this work.

The model. - We consider two incoherently interacting beams propagating along the direction $z$ in a bulk, weakly nonlinear saturable optical medium. The model corresponds, in the isotropic approximation, to the experimentally realized solitons in photorefractive materials. The problem is described by the normalized, coupled equations for the slowly varying beam envelopes, $E_{1}$ and $E_{2}$. The normalized dynamical equations for the envelopes of two incoherently interacting beams can, in this case, be approximately written in the form

$$
i \frac{\partial E_{1,2}}{\partial z}+\Delta_{\perp} E_{1,2}-\frac{E_{1,2}}{1+\left|E_{1}\right|^{2}+\left|E_{2}\right|^{2}}=0,
$$

where $\Delta_{\perp}$ is the transverse Laplacian. Stationary solutions of Eq. (1) can be found in the form $E_{1}=$ $\sqrt{\beta_{1}} u(x, y) \exp \left(i \beta_{1} z\right), \quad E_{2}=\sqrt{\beta_{1}} v(x, y) \exp \left(i \beta_{2} z\right)$, where $\beta_{1}$ and $\beta_{2}$ are two independent propagation constants. Measuring the transverse coordinates in the units of $\sqrt{\beta_{1}}$, and introducing the ratio of the propagation constants, $\quad \lambda=\left(1-\beta_{2}\right) /\left(1-\beta_{1}\right)$, from Eq. (1) we derive a system of stationary equations for the normalized envelopes $u$ and $v$ :

$$
\begin{aligned}
\Delta_{\perp} u-u+u f(I) & =0, \\
\Delta_{\perp} v-\lambda v+v f(I) & =0,
\end{aligned}
$$

where $f(I)=I(1+s I)^{-1}, I=u^{2}+v^{2}$, and $s=1-$ $\beta_{1}$ plays the role of a saturation parameter. For $s=0$, this system describes the Kerr nonlinearity. In this paper we will work with intermediate values of saturation, about $s=0.5$.

Vortex solitons. - We look for radially symmetric solutions $u(x, y)=u(r), v(x, y)=v(r) \exp (\operatorname{im} \phi)$, in which the second component carries a topological charge, $m$, and we assume that the $u$ component has no charge. In this case, Eqs. (2) take the form:

$$
\begin{aligned}
\Delta_{r} u-u+u f(I) & =0, \\
\Delta_{r} v-\left(m^{2} / r^{2}\right) v-\lambda v+v f(I) & =0,
\end{aligned}
$$

where $\Delta_{r}=(1 / r)(d / d r)(r d / d r)$. The fundamental, bell-shaped solutions with $m=0$ exist only at $\lambda=1$. In the remaining region of the parameter plane $(s, \lambda)$ the solutions carrying a topological charge $(m= \pm 1)$ in the second component do exist. This type of solution for the saturable nonlinearity is found and thoroughly investigated in [8] (and [7] for the so-called threshold nonlinearity). The families of these radially symmetric, two-component vector solitons are characterized by a single parameter $\lambda$, and, at any fixed value of $s$, the border of their existence domain is determined by a cutoff value, $\lambda_{c}$. A two-component trapped state exists only for $\lambda>\lambda_{c}$. Near the cutoff point, this bell-shaped state can be presented as a waveguide created by the $u$ component guiding a small-amplitude mode $v$. Away from the cutoff line, the amplitude of the $v$ component grows, and the resulting vector soliton develops a ringlike shape. An example of the ring-shaped vortex mode is presented in Fig. 2(b). An important physical characteristic of vector solitons of this type is the total power defined as $P=P_{u}+P_{v}=2 \pi \int_{0}^{\infty}\left(u^{2}+v^{2}\right) r d r$, where the partial powers $P_{u}$ and $P_{v}$ are the integrals of motion for the model (1). The dependencies $P(\lambda), P_{v}(\lambda)$, and $P_{u}(\lambda)$ for a fixed $s$ completely characterize the family of vector solitons [a typical example is shown in Fig. 2(a)] for $\lambda>\lambda_{c}$.
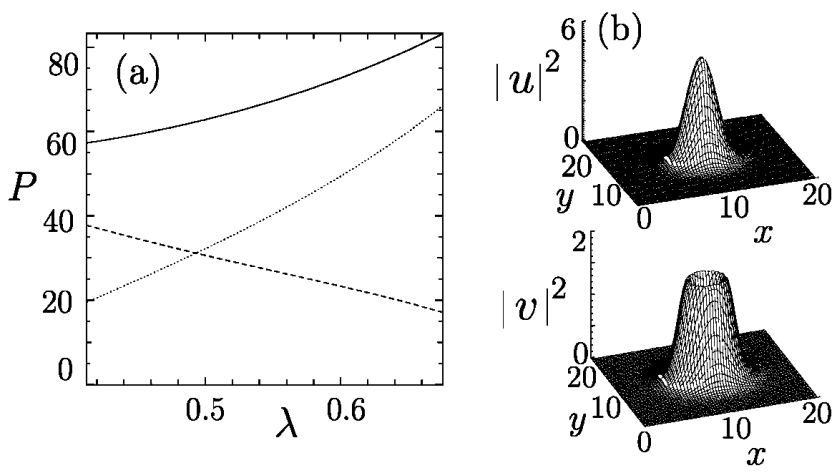

FIG. 2. (a) Power diagram for the dipole-mode soliton solution for $s=0.5$ (solid, total power; dashed, $P_{u}$; dotted, $P_{v}$ ) and (b) typical intensity profile of the $u$ (top) and $v$ (bottom) components $(\lambda=0.65)$. 
Stability analysis. - Similar to the well-studied case of the $(1+1)$-D vector solitons (see, e.g., Ref. [6]), the vortex-mode solitons are associated, in the linear limit, with a soliton-induced waveguide supporting a higher-order mode. It is therefore tempting to draw the analogy between the higher-order $(1+1)$-D two-hump solitons and $(2+1)$-D ring-shaped solitons. Given the established stability of the multihump one-dimensional structure in a saturable medium [6], this line of thought would lead us (erroneously) to conclude that the vortexmode vector solitons in our model should be stable.

To prove this prediction wrong, we have performed a linear stability analysis of the two-dimensional vortex-mode solitons. Our technique consists of linearizing the evolution equations (1) around the vortex solution, choosing a large set of completely random initial states and evolving them numerically using a split-step operator technique on a discrete Fourier basis. Typically, the solution will be a linear combination of modes which evolves with some real frequencies, $\mu$. However, if the linear equation bears modes with complex eigenvalues, $\mu=a+i b$, we expect an exponential growth of our random data, with convergence to the invariant space of one of such eigenvalues.

Our linear stability analysis has proved that, although saturation does have a strong stabilizing effect on the ring vector solitons [8], all vector solitons of this type are linearly unstable. As can be seen from Fig. 3(a), where we plot a typical dependence of the eigenvalue $\mu$ of the most unstable mode on the soliton parameter $\lambda$ for a fixed $s$, the growth rate of the instability, $\operatorname{Im}(\mu)$, tends to zero at the cutoff point of the vortex mode [cf. Fig. 2(a)]. This behavior is consistent with the inherent stability of the fundamental scalar soliton in a saturable medium. Elsewhere the growth rate is positive and its value increases when the intensity of the vortex mode is increased [cf. Figs. 2 and 3(a)].
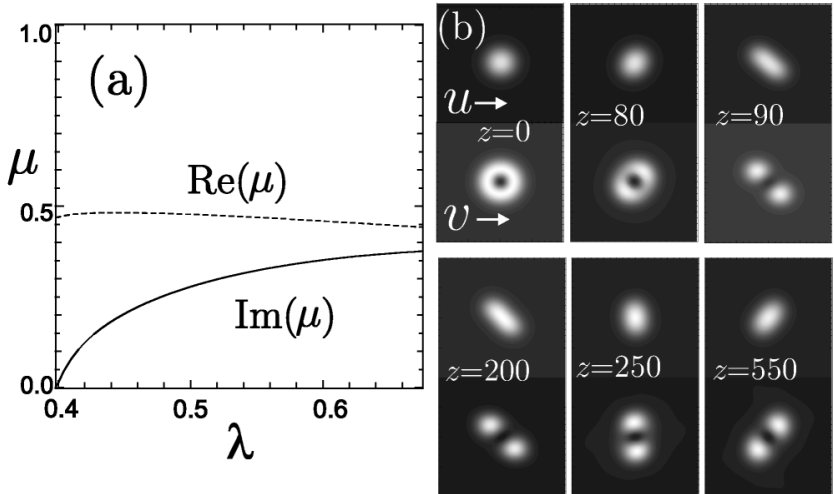

FIG. 3. (a) Eigenvalue of the leading unstable mode for vortexmode solution $(s=0.5)$ and (b) typical evolution of the vortex-mode soliton near cutoff $(s=0.65, \lambda=0.6)$. Shown are intensity distributions of the $u$ and $v$ components in the $(x, y)$ plane $[-10<(x, y)<10]$.
In Figs. 3(a) and 3(b) we compare the linear stability analysis with dynamical simulations of the vortex-mode soliton near cutoff, perturbed with a random noise. The instability, although largely suppressed by saturation, triggers the decay of the system into a dipole structure [as shown in Fig. 3(b)] for even a small contribution of the charged mode. The dipole demonstrates astonishing persistence for large propagation distances as a rotating and radiating pulsar state.

Dipole vector solitons. - It is apparent from the above analysis that the dipole-mode vector soliton should be more stable than the vortex-mode soliton. We have identified the existence domain of these solitons by solving numerically the stationary equations (2). One characteristic example of the dipole-mode family is shown in Fig. 4(a) for a fixed $s$. Our linear stability analysis for these solutions does not converge to a particular value of $\operatorname{Im}(\mu)$. This indicates that either the eigenvalues of the unstable modes, if they exist, are very small or the unstable modes have shapes which are only too weakly excited by random perturbations.

To obtain further information on the dynamical stability of dipole-mode vector solitons we have propagated numerically different perturbed dipole-mode solitons for distances up to several hundreds of $z$ units, or diffraction lengths. To put these numbers into physical perspective, we note that, in current experiments on solitons in photorefractive materials, the typical crystal length is $\approx 20 \mathrm{~mm}$, whereas $z=100$ in our model corresponds to the soliton propagation length $\approx 20-40 \mathrm{~mm}$.

We have performed two types of numerical experiments. We have found that small perturbations or random noise lead to bounded oscillations of the vector soliton, which retains its shape. This shows that the dipole-mode vector solitons should be stable enough for experimental observation. On the other hand, strong perturbations, such as a strong disproportion of the humps of the dipole or a relative displacement of the components, may alter the shape of the dipole. Such a perturbation, in the process of evolution, is typically transferred from one component to
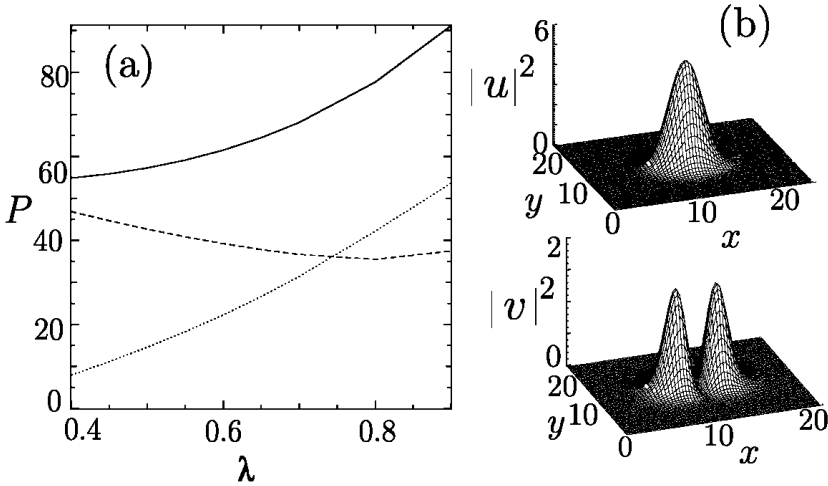

FIG. 4. (a) Power diagram of the dipole-mode soliton family $(s=0.5)$ and (b) typical intensity of the $u$ (top) and $v$ (bottom) components $(\lambda=0.65)$. 


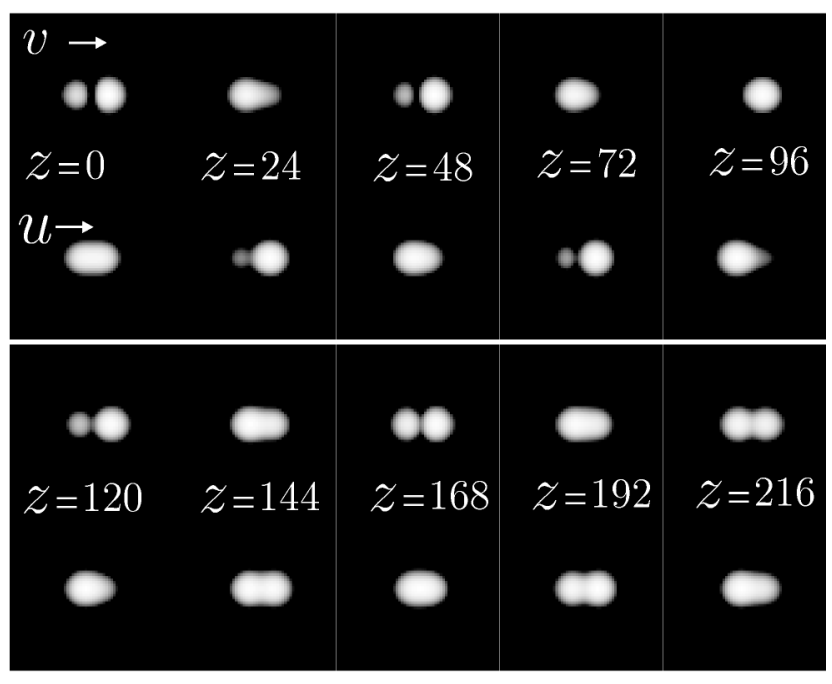

FIG. 5. Numerical evolution of a dipole vector soliton, $(\lambda, s)=(0.7,0.5)$, after moving $25 \%$ of the total power from one hump to another. Shown are intensity distributions for the $u$ and $v$ modes in the $(x, y)$ plane $[-7<(x, y)<7]$.

the other in a robust, periodic way. It is apparent from our simulations [Fig. 5] that in this case the dynamics is similar to two beams spiraling together with initial zero angular momentum [9].

From the evidence above it is clear that the unstable modes of a dipole-mode vector soliton, if they exist, should be rare and hard to excite. Calculations of the linear stability spectrum around the exact soliton solution should provide a complete answer to the stability question, which will be the subject of further research.

By summing up the stability results, we can state that the dipole-mode vector solitons are extremely robust objects. According to our numerical tests, they have a typical lifetime of several hundred diffraction lengths and survive a wide range of perturbations. This is true even for vector states with a large fraction power in the dipole component $\left(P_{v}>P_{u}\right)$, i.e., in the parameter region which cannot be safely reached for vortex-mode solitons, since the latter become unstable much sooner.

Finally, it is important to mention that there exist other physical models, where stable, radially asymmetric, dipole solitary waves play an important role in the nonlinear wave dynamics. All of these models, however, consider scalar, or one-component, structures. The most famous examples are the Larichev-Reznik soliton, a localized solution of the Charney equation for the Rossby waves [11], and the dipole Alfvén vortex soliton in an inhomogeneous plasma [12]. Another type of dipole solitary wave is found as a two-soliton bound state in a nonlocal [13] or anisotropic nonlocal [14] media due to anomalous interaction of two solitons with opposite phases. Nevertheless, the physics behind all known dipole solitary waves and corresponding single-component nonlinear models differs drastically from the problem considered above. Therefore, the dipole-mode vector soliton we describe in this Letter is a genuinely new type of solitary wave in a homogeneous bulk medium, a phenomenon that may occur in many other physical applications.

In conclusion, we have analyzed the existence and stability of radially symmetric and asymmetric higherorder vector optical solitons in a saturable nonlinear bulk medium, and predicted a new type of optical soliton that is associated with a dipole mode guided by the soliton-induced waveguide. We have demonstrated that the solitons carrying a topological charge are unstable and, as a consequence, they may decay into dipole-mode solitons. There is also strong evidence of the stability of these dipole-mode solitons. We believe that all of the effects predicted in this Letter, including the existence and stability of dipole-mode solitons, can be easily verified in experiments with photorefractive media.

J. J. G-R. thanks the Optical Sciences Center, ANU, for warm hospitality during his stay in Australia. V.M. P-G. and J.J.G-R. are partially supported by DGICYT under Grant No. PB96-0534.

[1] See, e.g., G. I. Stegeman and M. Segev, Science 286, 1518 (1999), and references therein.

[2] D. N. Christodoulides and R. J. Joseph, Opt. Lett. 13, 53 (1988).

[3] M. R. Matthews et al., Phys. Rev. Lett. 83, 2498 (1999); J. J. García-Ripoll and V. M. Pérez-García, Phys. Rev. Lett. 84, 4264 (2000); J. Williams and M. Holland, Nature (London) 401, 568 (1999).

[4] Th. Busch and J.R. Anglin, Phys. Rev. A 60, R2669 (1999); J. J. García-Ripoll et al., Phys. Rev. A 61, 053609 (2000).

[5] See, e.g., M. San Miguel, Phys. Rev. Lett. 75, 425 (1995); M. Hoyuelos et al., Comput. Phys. Commun. 121, 414 (1999); M. Hoyuelos et al., Phys. Rev. E 58, 2992 (1998).

[6] E. A. Ostrovskaya, Yu. S. Kivshar, D. V. Skryabin, and W. Firth, Phys. Rev. Lett. 83, 296 (1999).

[7] Z.H. Musslimani, M. Segev, D. N. Christodoulides, and M. Soljačić, Phys. Rev. Lett. 84, 1164 (2000).

[8] J. N. Malmberg, A. H. Carlsson, D. Anderson, M. Lisak, E. A. Ostrovskaya, and Yu. S. Kivshar, Opt. Lett. 25, 643 (2000).

[9] A. V. Buryak, Yu. S. Kivshar, M. Shih, and M. Segev, Phys. Rev. Lett. 82, 81 (1999).

[10] D. N. Christodoulides et al., Appl. Phys. Lett. 68, 1763 (1996); Z. Chen et al., Opt. Lett. 21, 1436 (1996).

[11] V. D. Larichev and G. M. Reznik, Dokl. Akad. Nauk SSSR 231, 1077 (1976) [Sov. Phys. Dokl. 21, 581 (1976)].

[12] V. I. Petviashvili and O. A. Pokhotelov, Pis'ma Zh. Eksp. Teor. Fiz. 42, 47 (1985) [JETP Lett. 42, 54 (1985)].

[13] V. A. Mironov et al., [Sov. Phys. Dokl. 25, 861 (1981)]. Dokl. Akad. Nauk SSSR, 260, 325 (1981).

[14] A. V. Mamaev, A.A. Zozulya, V. K. Mezentsev, D. Z. Anderson, and M. Saffman, Phys. Rev. A 56, R1110 (1997). 